\title{
CARACTERIZAÇÃO E SIGNIFICADO PALEOAMBIENTAL DA FAUNA DE OSTRACODES DA FORMAÇÃO CODÓ (NEOAPTIANO), LESTE DA BACIA DE GRAJAÚ, MA, BRASIL
}

\author{
MARIA INES FEIJÓ RAMOS \\ MPEG, CPPG, Av. Perimetral, 1901, Terra Firme, Cx. P. 399, 66077-530, Belém, PA, Brasil.mramos@museu-goeldi.br \\ DILCE DE FÁTIMA ROSSETTI \\ INPE, Rua dos Astronautas, 1758, Jardim da Granja, Cx. P. 515, 12245-970, São José dos Campos, SP, Brasil.rossetti@dsr.inpe.br
}

JACKSON D. S. PAZ

MPEG, CPPG, Av. Perimetral, 1901, Terra Firme, Cx. P. 399, 66077-530, Belém, PA, Brasil. jackdspaz@yahoo.com.br

\begin{abstract}
RESUMO - O estudo de ostracodes provenientes de seções estratigráficas expostas na região leste da bacia de Grajaú, Estado do Maranhão, referente à Formação Codó, revelou a presença de uma fauna neoaptiana tipicamente lacustre composta exclusivamente por Candona e Harbinia, representada pelas espécies Candona sp., Harbinia micropapillosa (Bate), Harbinia angulata (Krömmelbein \& Weber), Harbinia sinuata (Krömmelbein \& Weber), Harbinia sp. aff. H. symmetrica (Krömmelbein \& Weber), Harbinia salitrensis (Krömmelbein \& Weber) e Harbinia sp. O padrão de distribuição estratigráfica das paleocomunidades de ostracodes nas seções estudadas confirmou a ocorrência de uma ambiente deposicional lacustre e cíclico, como inferido por estudos faciológicos anteriores. A ciclicidade do sistema está relacionada a eventos de contração e expansão do lago caracterizado predominantemente como hipersalino, de águas relativamente rasas e com pouca disponibilidade de oxigênio. As bruscas mudanças ambientais causadas por estes eventos proporcionaram a ocorrência monoespecífica de algumas espécies mais resistentes, seguida de mortandade em massa.
\end{abstract}

Palavras-chave: Ostracodes lacustres, Neoaptiano, Formação Codó, bacia de Grajaú, Maranhão.

\begin{abstract}
IDENTIFICATION AND PALEOENVIRONMENTAL SIGNIFICANCE OF THE OSTRACOD FAUNA FROM CODÓ FORMATION (NEOAPTIAN), EAST OF THE GRAJAÚ BASIN, MA, BRAZIL. The study of ostracods from sedimentary successions exposed in the east portion of the Grajau Basin, Maranhão State, related to Codó Formation, revealed a Neoaptian lacustrine fauna characterized exclusively by Harbinia and Candona represented by the species Candona sp., Harbinia micropapillosa (Bate), Harbinia angulata (Krömmelbein \& Weber), Harbinia sinuata (Krömmelbein \& Weber), Harbinia sp. aff. H. symmetrica (Krömmelbein \& Weber), Harbinia salitrensis (Krömmelbein \& Weber) and Harbinia sp. The stratigraphical distribution patterns of the ostracod palaeocommunities in the studied sections confirmed the occurrence of a cyclic depositional lacustrine environment, as assumed by previous faciological studies. These cycles are associated with events of expansion and contraction of the lake, characterized mainly as hipersaline, with shallow-water and very low oxygen levels. The sudden environmental changes caused by these events produced a monospecific occurrence of some resilient species, followed by a mass death.
\end{abstract}

Key words: Lacustrine ostracods, Neoaptian, Codó Formation, Grajaú Basin, Maranhão.

\section{INTRODUÇÃO}

O domínio de um ambiente de sedimentação lacustre com pouca disponibilidade de oxigênio faz com que a Formação Codó seja uma unidade de interesse paleontológico pelo grande potencial de preservação de fósseis. Apesar disto, não há ainda estudos suficientes que permitam caracterizar adequadamente o conteúdo fossilífero desta unidade. Um bom exemplo da falta de informações paleontológicas referentes a estes depósitos pode ser ilustrado pelos ostracodes, cujos registros se restringem a uma breve menção em estudos sedimentológicos anteriores (Paz \& Rossetti, 2001, 2005) e a um único trabalho relacionado a estes microfósseis (Krömmelbein \& Weber,1971), ficando sua sistemática e aplicação a interpretação paleoambiental ainda por ser documentada. 
Nos últimos anos, diversos estudos enfocando análises faciológicas, estratigráficas e geoquímicas foram realizados nestes depósitos, visando a sua reconstituição paleoambiental (Rossetti \& Góes, 2000; Paz \& Rossetti, 2001, 2005; Rossetti et al., 2004 e Paz, 2005). Como resultado, houve a proposição de um sistema deposicional lacustre, hipersalino, dominantemente anóxico e sujeito a flutuações periódicas da lâmina de água, característica esta revelada pela organização faciológica em ciclos de arrasamento ascendentes (Paz \& Rossetti, 2005).

Este trabalho tem por objetivos descrever os ostracodes provenientes de um afloramento exposto na borda leste da bacia do Grajaú, no Estado do Maranhão, referente à Formação Codó e auxiliar no refinamento das interpretações paleoambientais. O estudo de ostracodes aqui apresentado disponibilizará informações adicionais que contribuirão para o refinamento das interpretações paleoambientais vigentes.

\section{MATERIAL E MÉTODOS}

O material de estudo é proveniente de diversas coletas realizadas em uma mina de calcário na localidade de Santo Amaro (Figura 1), ambas no município de Codó, leste do Estado do Maranhão. As amostras examinadas foram processadas no Laboratório de Sedimentologia do Museu Paraense Emílio Goeldi (MPEG) onde foram, primeiramente, fragmentadas em pequenas partes, e posteriormente, submersas em água para desagregação. Quando necessário, as amostras foram tratadas com ácido clorídrico, para melhor desagregação e recuperação dos ostracodes. Posteriormente, as amostras foram lavadas e peneiradas $(0,32 ; 0,117$ e $0,065 \mathrm{~mm})$ por métodos convencionais, com posterior triagem e identificação dos espécimes. As fotografias foram obtidas no MEV do Laboratório de Microscopia Eletrônica (LME) do MPEG. Os espécimes figurados encontramse depositados na Coleção Paleontológica do MPEG, Seção de Micropaleontologia, sob os números MPEG-31-MO a MPEG56-MO. A descrição sistemática aqui apresentada segue Moore (1961) e a literatura citada.

\section{CONTEXTO GEOLÓGICO}

A bacia de Grajaú, localizada em grande parte do Estado do Maranhão (Figura 1), separa-se da bacia paleozóica do Parnaíba, a sul, pelo Lineamento Estrutural Xambioá(Góes \& Coimbra, 1996; Góes \& Rossetti, 2001), a leste pelo Lineamento do Rio Parnaíba e a noroeste pelo Arco do Capim; está conectada, a norte, à bacia de São Luís, com a qual demonstra continuidade física pelo menos a partir do final do Cretáceo. A gênese desta bacia acha-se ligada a cisalhamento puro e transcorrência, associados à instalação de sistema rift intracontinental abandonado (Azevedo, 1991; Góes \& Rossetti, 2001) que se desenvolveu ao longo da margem equatorial brasileira durante a separação dos continentes africano e sul-americano, no Neojurássico/Eocretáceo.

O preenchimento sedimentar da bacia de São Luís-Grajaú consiste em rochas cretáceas e em uma delgada cobertura cenozóica (Figura 2). O estágio pré-rift ocorreu no Neoaptino, sendo registrado por folhelhos negros, evaporitos e calcários lacustres da Formação Codó, além de depósitos flúvio-deltaicos da Formação Grajaú. Estes estratos são recobertos por uma espessa sucessão sedimentar formada a partir do Albiano, inserida no Grupo Itapecuru (Rossetti \& Truckenbrodt, 1997), o qual inclui a Unidade Indiferenciada (Eo/Mesoalbiano), a Formação Alcântara (Neoalbiano-Cenomaniano), e a Formação

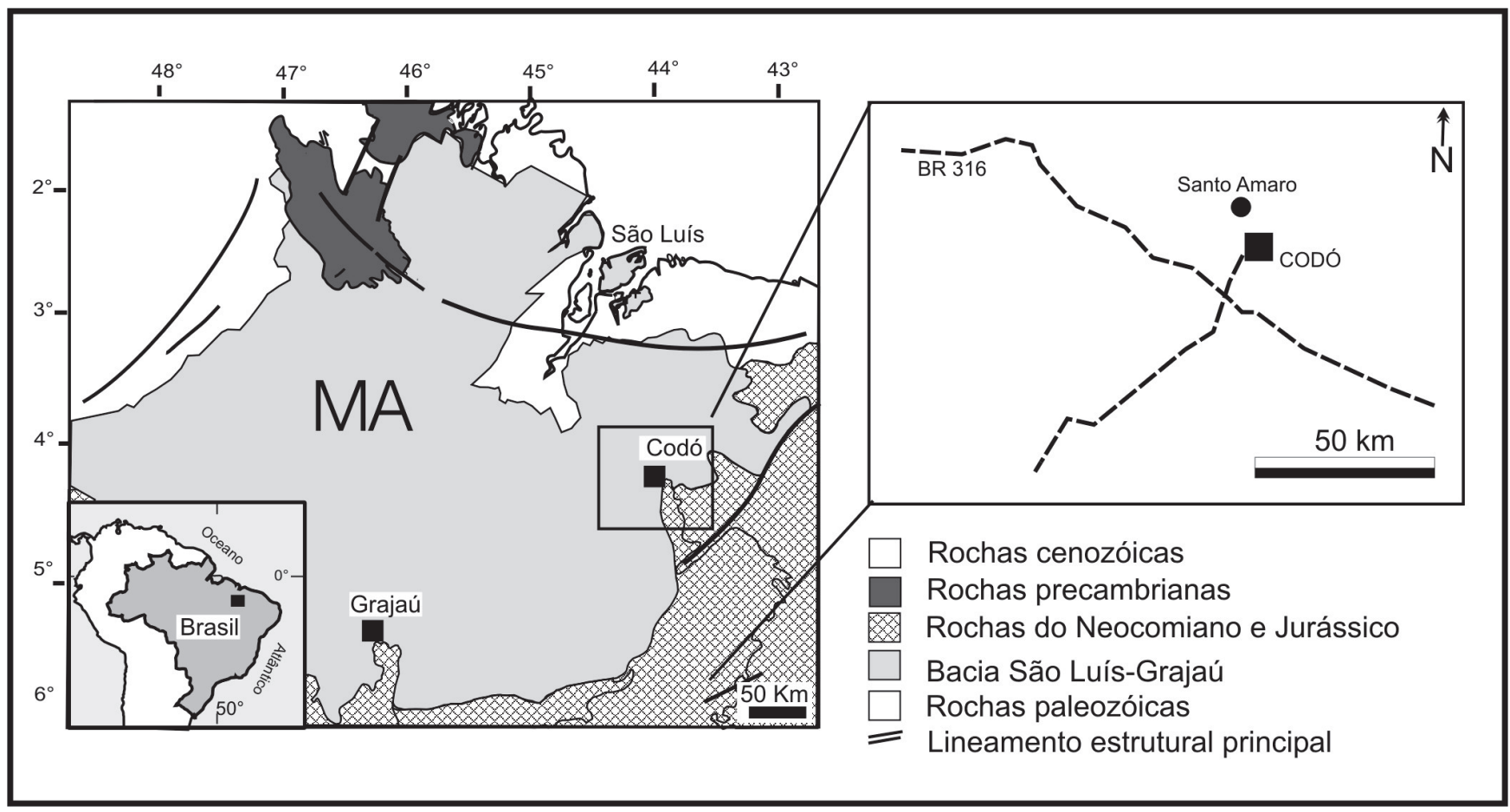

Figura 1. Mapa de localização da bacia de Grajaú e da área de estudo (modificado de Paz \& Rossetti, 2001).

Figure 1. Location map of the Grajaú Basin and the studied area (modified from Paz \& Rossetti, 2001). 
Cujupe (Cretáceo Superior). A Unidade Indiferenciada ocorre principalmente em subsuperfície e consiste em uma sucessão de aproximadamente $1.500 \mathrm{~m}$ de espessura. Em superfície, estes depósitos são dominantemente deltaico-lagunares, com retrabalhamento por ondas normais e de tempestade (Miranda, 2005). As formações Alcântara e Cujupe são bem representadas em afloramentos na porção central e norte da bacia, e incluem uma variedade de depósitos areníticos e pelíticos e, menos comumente, carbonáticos, que foram formados sob influência de processos ligados a ondas e correntes de maré, sendo atribuídos a ambientes de ilha-barreira e estuário, respectivamente (Rossetti, 1997a, 1997b, 1998). A sucessão cenozóica da bacia é excepcionalmente bem representada em afloramentos, sendo em grande parte de idade miocênica. Estes estratos são principalmente de estuários e mistos carbonático-siliciclásticos, que evoluíram para exclusivamente siliciclásticos para o topo, sendo correspondentes aos termos litoestratigráficos Pirabas/ Barreiras Inferior, Barreiras Médio/Superior e Barreiras Superior (Rossetti, 2000, 2001).

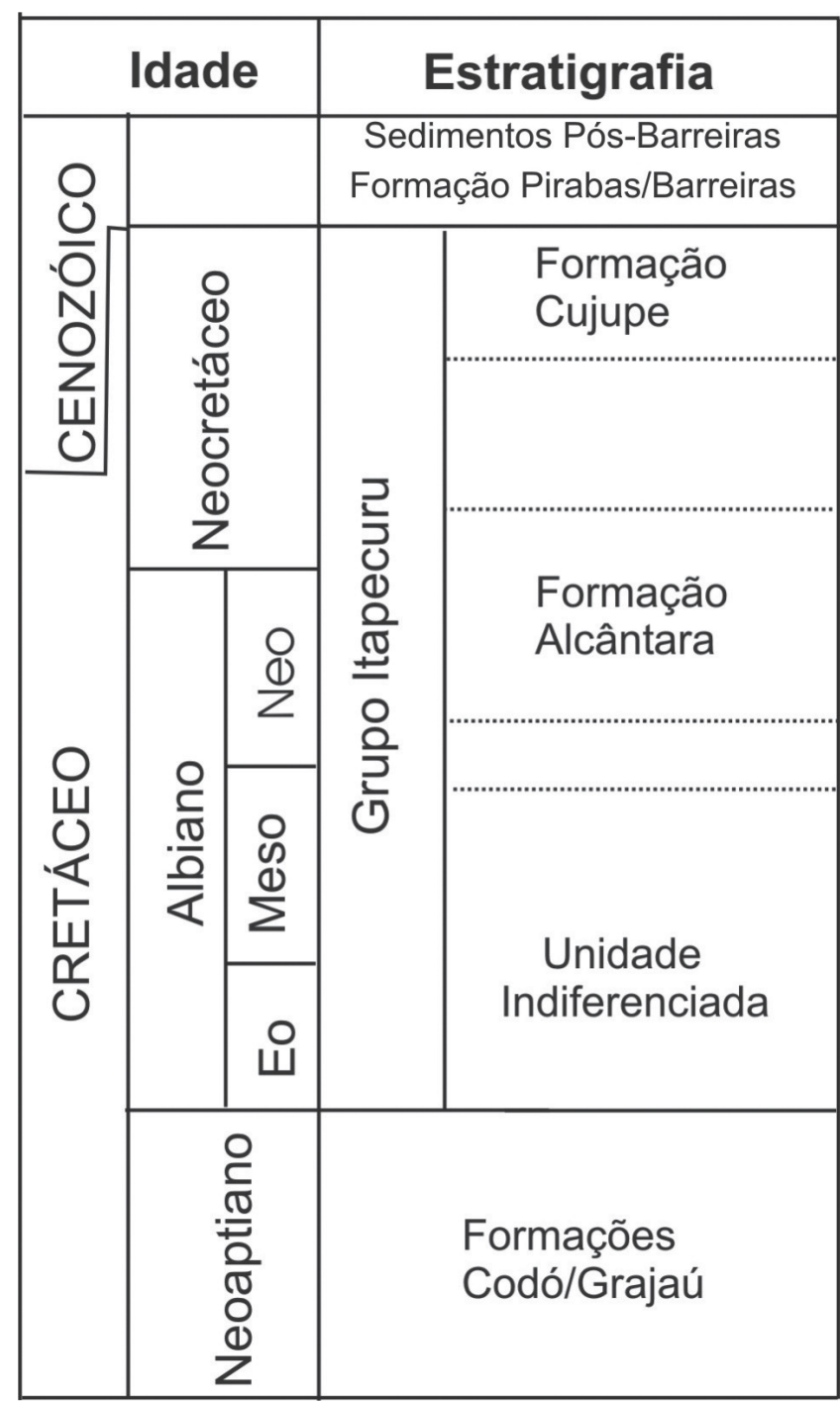

Figura 2. Coluna estratigráfica da bacia de São Luís-Grajaú (adaptado de Rossetti, 2001).

Figure 2. Stratigraphic chart of São Luís-Grajaú Basin (adapted from Rossetti, 2001).

\section{Caracterização da Formação Codó na área de estudo}

A caracterização sedimentológica da Formação Codó exposta na área de estudo já foi apresentada na literatura (Paz, 2005; Paz \& Rossetti, 2001, 2005), sendo sumarizada aqui a fim de contextualização e integração com os dados de ostracodes apresentados neste trabalho. Esta unidade consiste em três associações faciológicas, atribuídas a ambientes de lago central, lago transicional e lago marginal. A associação de fácies de lago central é constituída por pacotes de até $10 \mathrm{~m}$ de espessura de folhelho negro e evaporito, os quais compõem a base de ciclos de arrasamento ascendente. O folhelho negro é betuminoso, rico em ostracodes e, localmente, com pirita e enxofre nativo. $\mathrm{O}$ evaporito é constituído de gipso nodular, acicular, fibroso e radial e, subordinadamente, anidrita. A associação de fácies de lago transicional, posicionada entre as associações de fácies de lago central e lago marginal, é constituída de argilito laminado ou interacamadados de argilito laminado e calcário com até $5 \mathrm{~m}$ de espessura. A associação de fácies de lago marginal compreende pacotes com até 7,5 $\mathrm{m}$ de espessura, sendo representada por pelito maciço, calcita-arenito, gipso-arenito, calcário ostracodal, calcário pisoidal, tufa e ritmito calcário/folhelho. Estes depósitos apresentam horizontes de paleossolo, carstificação, fenestra, além de marcas de ondas normais (Figura 3).

Os depósitos acima descritos foram atribuídos a sistema deposicional dominantemente lacustre, tendo-se em vista as características faciológicas com domínio de litologias formadas por suspensão, ausência de fósseis marinhos, e dados isotópicos de ${ }^{87} \mathrm{Sr} /{ }^{86} \mathrm{Sr}$ e ${ }^{34} \mathrm{~S} /{ }^{32} \mathrm{~S}$ que não correspondem àqueles típicos de águas marinhas durante o Aptiano (Paz, 2005; Rossetti et al., 2000; Rossetti et al., 2004). Estes estratos são organizados em ciclos de arrasamento ascendentes atribuídos à progradação de ambientes marginais sobre ambientes centrais, em sistema lacustre. Dados faciológicos mostram que o paleolago era caracterizado por uma bacia hidrologicamente fechada, dominantemente anóxico, sem aporte de grãos terrígenos grossos, hipersalino e com lâmina de água relativamente rasa (Paz, 2005).

A análise palinológica da Formação Codó na área de estudo levou ao estabelecimento de idade neoaptiana com base na presença de Sergipea variverrucata (Paz, 2005; Rossetti et al., 2000).

\section{SISTEMÁTICA PALEONTOLÓGICA}

Classe OSTRACODA Latreille, 1806

Ordem PODOCOPIDA Muller, 1894

Superfamília CYPRIDACEA Baird, 1845

Família CYPRIDIDAE Baird, 1845

Subfamília CYPRIDINAE Baird, 1845

Gênero Harbinia (Tsao, 1959) Hou, 1984

Harbinia micropapillosa (Bate, 1972)

(Figura 4A-D)

Pattersoncypris micropapillosa Bate,1972:379A-393, pls. 66-71. Pattersoncypris micropapillosa Bate,1973:101-108, pls.1:19:102 e 1:19:108. 


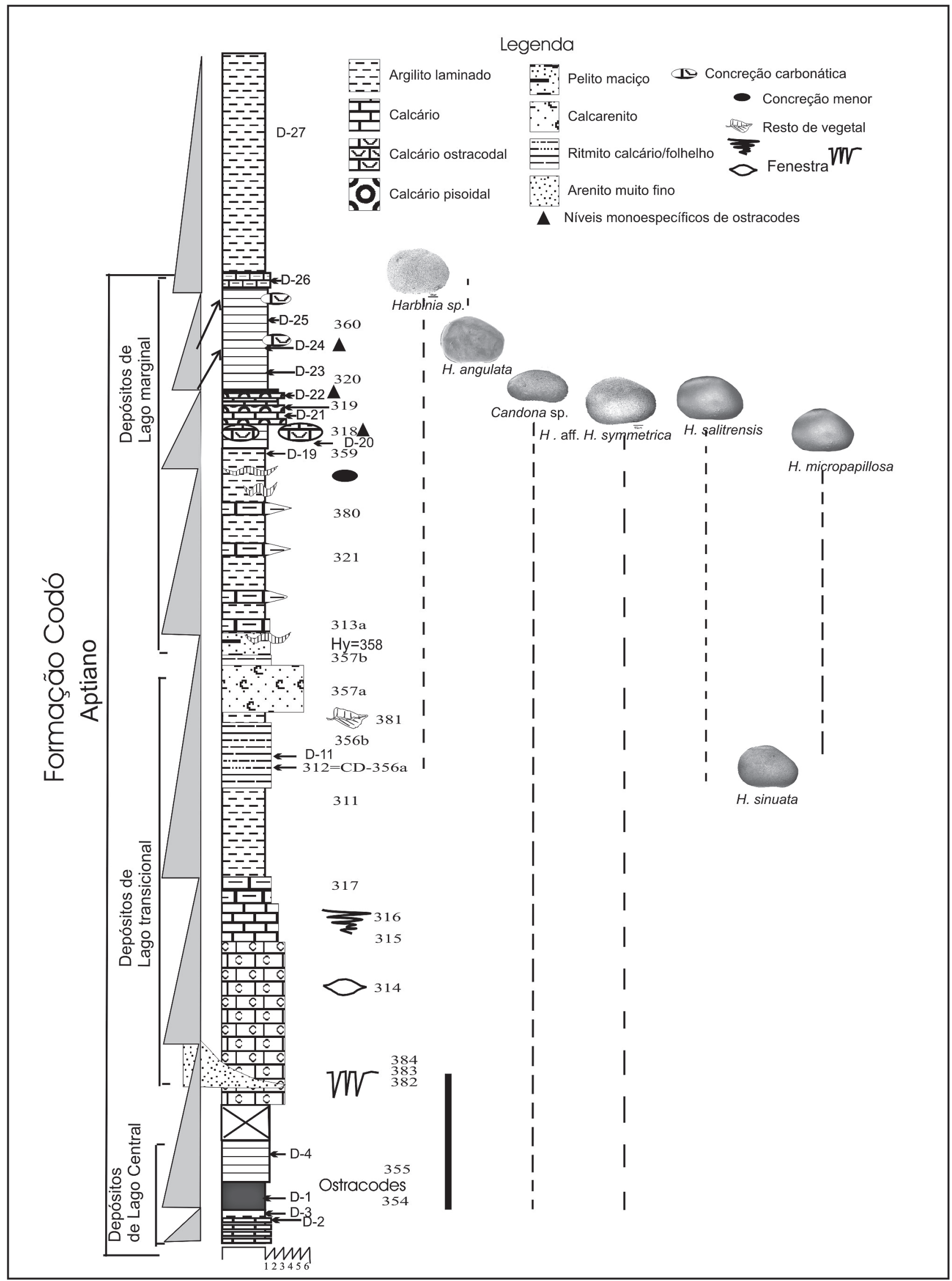

Figura 3. Perfil litoestratigráfico da localidade de Santo Amaro, com indicação do posicionamento estratigráfico das amostras estudadas e a distribuição das espécies de ostracodes.

Figure 3. Lithostratigraphic profile of Santo Amaro locality indicating the studied samples position and ostracod species distribution. 
Hourcqia angulata angulata (Krömmelbein \& Weber, 1971).Viana, Brito \& Silva-Telles, 1989:216, figs. 2a, b, c.

Hourcqia angulata angulata (Krömmelbein \& Weber, 1971). Depeche, Berthou \& Campos, 1990:303-304, pl. 1, figs. 1-2.

[Non] Pattersoncypris micropapillosa Bate, 1972. Depeche, Berthou \& Campos, 1990:305-306, pl. 3, figs. 6-7, 13.

Hourcqia angulata angulata (Krömmelbein \& Weber, 1971). Depeche, Berthou \& Campos, 1990:305-306, pl. 3, fig. 17.

Localidade tipo. Aptiano/Albiano, Formação Santana (bacia do Araripe), Ceará, norte do Brasil.

Espécimes figurados. MPEG-31-MO a MPEG-33-MO.

Dimensões. MPEG-31-MO, carapaça, adulto, valva esquerda, comprimento: $1,094 \mathrm{~mm}$; altura: $0,7422 \mathrm{~mm}$; valva direita, comprimento: 1,073 mm; altura: 0,719 mm;MPEG-32-MO, carapaça, juvenil, valva esquerda, comprimento: $0,7378 \mathrm{~mm}$; altura: 0,5195 mm; MPEG-33-MO, carapaça, adulto, valva esquerda, comprimento: $1,068 \mathrm{~mm}$; altura: $0,719 \mathrm{~mm}$; largura: $0,559 \mathrm{~mm}$.

Localidade. Mina de Santo Amaro, Município de Codó, Estado do Maranhão.

Horizonte. Amostra CD-318, Aptiano Superior, Formação Codó, bacia de Grajaú.

Material. Abundante (aproximadamente 200 espécimes).

Ocorrência na área de estudo. Amostras CD-356a e CD-318.

Outras ocorrências. Formação Santana (bacia do Araripe) e Formação Alagamar (bacia Potiguar).

Discussão. A posição genérica de Harbinia micropapillosa (Bate, 1972) foi amplamente discutida por do Carmo et al. (2004), os quais, após a análise das espécies-tipo dos gêneros Hourcqia e Pattersoncypris constataram similaridades marcantes com o gênero Harbinia, considerando-os como sinonímia deste último. No presente trabalho segue-se a proposta de do Carmo et al. (2004) para as espécies anteriormente atribuídas a Pattersoncypris e Hourcqia considerando-se, assim, as espécies aqui identificadas como pertencentes ao gênero Harbinia. Os espécimes aqui identificados como Harbinia micropapillosa têm as mesmas características morfológicas apresentadas por Bate (1972). O estudo comparativo desta espécie com $H$. angulata angulata como registrado por outros autores, conforme lista sinonímica apresentada, permitiu verificar que as mesmas espécies têm sido consideradas erroneamente como tal; as ilustrações inadequadas destas espécies, bem como a falta de um estudo sistemático detalhado e a má preservação do material, levaram a identificações confusas em relação às espécies deste gênero. Verificou-se que $H$. micropapillosa apresenta uma característica diagnóstica distinta das demais espécies do mesmo gênero, quer seja a presença da região dorsomediana fortemente acentuada, formando uma corcova bem pronunciada; são ainda características de H. micropapillosa: forma mais triangular que retangular, região posterior angulosa e região ventral quase reta. Apesar de H. micropapillosa ter sido considerada por alguns autores (ver lista sinonímica) como H. angulata angulata, assemelha-se mais a Harbinia sinuata (Krömmelbein \& Weber, 1971). Esta última não apresenta a corcova dorso mediana tão acentuada e tem a região pósterodorsomediana fortemente declinada em direção à região póstero-ventromediana; além disso, a região ventral é bem mais côncava do que em $H$. micropapillosa. Cabe salientar que as espécies $H$. micropapillosa, $H$. angulata e Harbinia sp. aqui registradas apresentam ornamentação levemente micropapilosa ou com microretículo (Figuras 4C, E, V), não sendo esta uma característica específica de $H$. micropapillosa. A ausência de estruturas ornamentais em alguns espécimes provavelmente está associada a processos diagenéticos e à má preservação, com conseqüente perda destas estruturas.

\section{Harbinia angulata (Krömmelbein \& Weber, 1971)} (Figuras 4E, H)

Hourcqia angulata angulata Krömmelbein \& Weber, 1971:3435, taf. 6, fig. 23 a, b, c.

[Non] Harbinia angulata angulata (Krömmelbein \& Weber, 1971). Depeche, Berthou \& Campos, 1990:305-306, pl. 2, fig. 1. [Non] Harbinia angulata (Krömmelbein \& Weber, 1971). Depeche, Berthou \& Campos, 1990:307-308, pl.3, figs. 10-11. Hourcqia angulata angulata (Krömmelbein \& Weber, 1971). Silva-Telles \& Viana, 1990:318-321, est. 3; fig. 3.

Hourcqia angulata angulata (Krömmelbein \& Weber, 1971). Depeche, Berthou \& Campos, 1990:307-308, pl. 3, fig. 18. Harbinia angulata (Krömmelbein \& Weber, 1971). do Carmo, 1998:32, est. 4, figs. 2-5.

Localidade-tipo. Estado do Alagoas, Brasil, Aptiano, Série Pós-Bahia, Formação Riachuelo.

Espécimes figurados. MPEG-34-MO a MPEG-37-MO.

Dimensões. MPEG-34-MO, carapaça, juvenil, valva esquerda, comprimento: 0,776 mm; altura: 0,570 mm; MPEG-35-MO, carapaça, adulto, valva direita, comprimento: $0,811 \mathrm{~mm}$; altura: 0,528 mm; MPEG-36-MO carapaça, juvenil, valva esquerda, comprimento: 0,786 mm; altura: 0,537 mm; MPEG-37-MO, carapaça, juvenil, valva esquerda, comprimento: $0,789 \mathrm{~mm}$; largura: $0,443 \mathrm{~mm}$.

Localidade. Mina de Santo Amaro, Município de Codó, Estado do Maranhão.

Horizonte. Amostra D-24, Aptiano Superior, Formação Codó, bacia de Grajaú.

Material. Abundante (aproximadamente 250 espécimes).

Ocorrência na área de estudo. Amostras D-24 e 26.

Outras ocorrências. Formação Riachuelo, Série Pós-Bahia; Formação Santana (bacia do Araripe).

Discussão. Harbinia angulata (Krömmelbein \& Weber, 1971) difere de H. micropapillosa (Bate, 1972) e de H. sinuata (Krömmelbein \& Weber, 1971) por não apresentar a corcova dorso-mediana bem acentuada presente em H. micropapillosa, e pela forma mais ovalada a quase retangular, diferente da forma subtriangular das demais; apresenta, ainda, a região posterior quase reta e os ângulos ântero e póstero-dorsomediano menos evidentes que nas outras espécies. Além disso, os espécimens de $H$. angulata são menores que $H$. micropapillosa.

\section{Harbinia sinuata (Krommelbein \& Weber, 1971) (Figuras 4I-L)}

Hourcqia angulata sinuata Krommelbein \& Weber, 1971:3536, taf. 6, fig. 24 


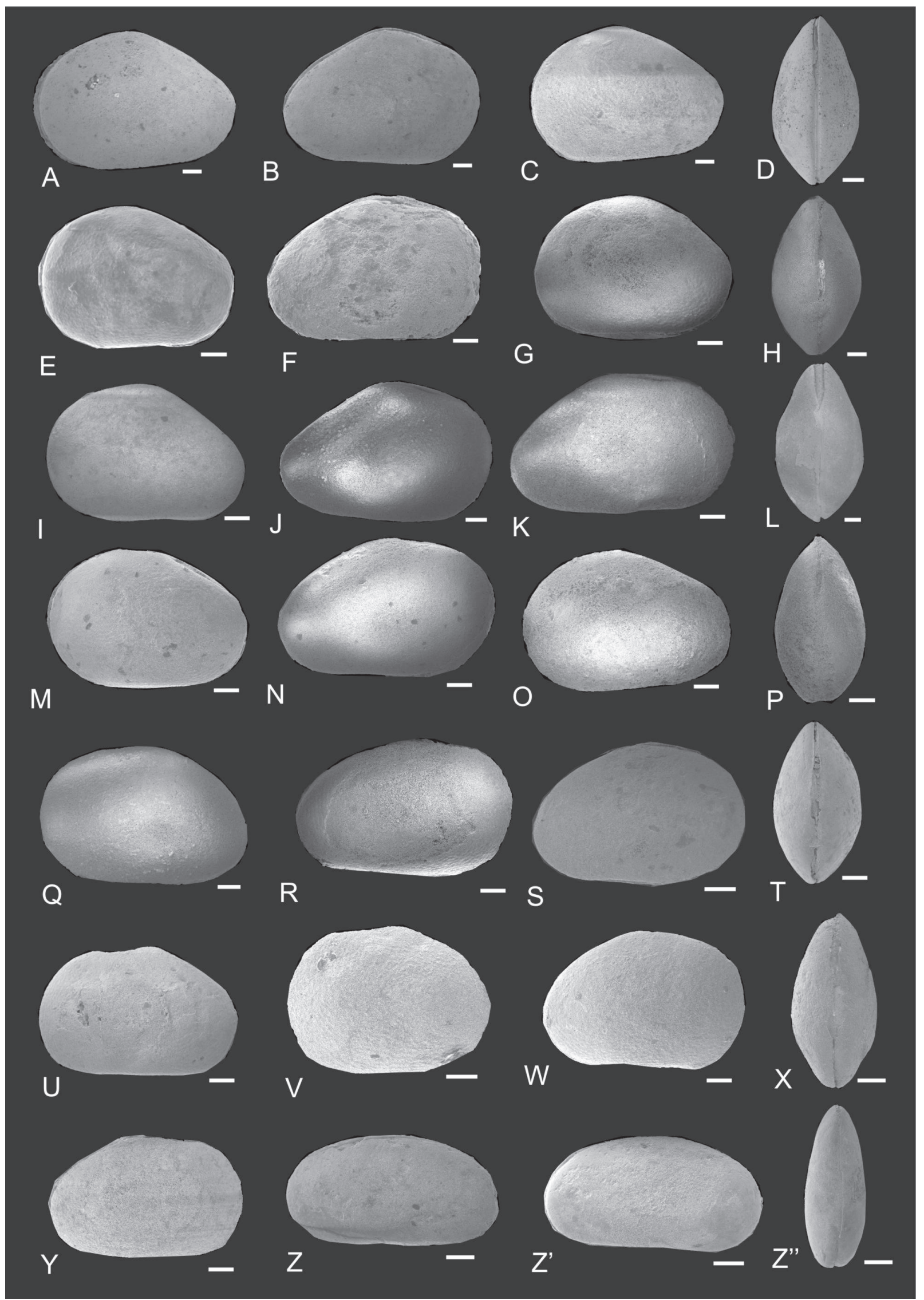

Figura 4. Ostracodes da bacia de Grajaú, Formação Codó. A-D, Carapaças de Harbinia micropapillosa: A, VE, MPEG-31-MO; B, VD, MPEG-31-MO; C, VE, juvenil, MPEG-32-MO; D, vista dorsal, MPEG-33-MO. E-H, Carapaças de Harbinia angulata: E, VE, juvenil, MPEG-34MO; F, VD, MPEG-35-MO; G, VE, juvenil, MPEG-36-MO; H, vista dorsal, juvenil, MPEG-37-MO). I-L, Carapaças de Harbinia sinuata: I, VE, 
Hourcqia angulata spp. Silva-Telles \& Viana, 1990:318-321, est. 3 , fig. 4 .

Hourcqia angulata (Krommelbein \& Weber, 1971). Depeche, Berthou \& Campos, 1990:305-308, pl. 2, figs. 4-5; pl. 3, figs. 8, 11 .

Harbinia sinuata (Krommelbein \& Weber, 1971). do Carmo, 1998:30, est. 2, figs. 1-5.

Localidade-tipo. Estado do Alagoas, Brasil, Aptiano, Formação Riachuelo, Série Pós-Bahia.

Espécimes figurados. MPEG-38-MO a MPEG-41-MO.

Dimensões. MPEG-38-MO, carapaça, juvenil, valva esquerda, comprimento: 0,796 mm; altura: 0,554 mm; MPEG-39-MO, carapaça, adulto, valva direita, comprimento: $1,042 \mathrm{~mm}$; altura: 0,682 mm; MPEG-40-MO carapaça, juvenil, valva esquerda, comprimento: 0,986 mm; altura: 0,617 mm; MPEG-41-MO, carapaça, juvenil, valva esquerda, comprimento: $0,990 \mathrm{~mm}$; largura: $0,557 \mathrm{~mm}$.

Localidade. Mina de Santo Amaro, Município de Codó, Estado do Maranhão.

Horizonte. Amostra CD-356a, Aptiano Superior, Formação Codó, bacia de Grajaú.

Material. Comum (aproximadamente 50 espécimes).

Ocorrência na área de estudo. Amostra CD-356a.

Outras ocorrências. Formação Riachuelo, Série Pós-Bahia; Formação Santana, bacia do Araripe; Formação Alagamar, bacia Potiguar.

Discussão. Harbinia sinuata (Krömmelbein \& Weber, 1971) é muito semelhante a $H$. micropapillosa diferindo desta por apresentar a corcova dorso-mediana menos acentuada, margem anterior quase reta, região dorso-posterior mais inclinada, além de apresentar a região ventral levemente côncava na região mediana.

Harbinia aff. H. symmetrica (Krommelbein \& Weber, 1971) (Figuras 4M-P)

Hourcqia angulata symmetrica Krommelbein \& Weber, 1971:36-37, est. 6, fig. 25a, b.

Hourcqia? sp. 2 Silva-Telles \& Viana, 1990:318-321, est. 2, fig. 8. Hourcqia angulata symmetrica (Krommelbein \& Weber, 1971). Silva-Telles \& Viana, 1990: 318-321, est. 1, e est. 3, fig. 8. Cultella sp.1 e sp.2. Depeche, Berthou \& Campos, 1990:305308, pl. 2, fig. 1-3.
Pattersoncypris micropapillosa (Bate, 1972). Depeche, Berthou \& Campos, 1990:307-308, pl. 3, fig. 7.

Cultella sp. Depeche, Berthou \& Campos, 1990:307-308, pl. 3, fig. 19.

Localidade tipo. Perfuração VGST-1-MA (384 m), Estado do Maranhão, Brasil, Aptiano, Formação Codó, bacia do Maranhão.

Espécimes figurados. MPEG-42-MO a MPEG-45-MO.

Dimensões. MPEG-42-MO, carapaça, juvenil, valva esquerda, comprimento: 0,784 mm; altura: 0,545 mm; MPEG-43-MO, carapaça, adulta, valva direita, comprimento: $0,954 \mathrm{~mm}$; altura: 0,603 mm; MPEG-44-MO carapaça, juvenil, valva esquerda, comprimento: 0,785 mm; altura: 0,530 mm; MPEG-45-MO, carapaça, juvenil, valva esquerda, comprimento: $0,686 \mathrm{~mm}$; largura: $0,375 \mathrm{~mm}$.

Localidade. Mina de Santo Amaro, Município de Codó, Estado do Maranhão.

Horizonte. Amostra CD-356a, Aptiano Superior, Formação Codó, bacia de Grajaú.

Material. Comum (aproximadamente 40 espécimes).

Ocorrência naárea de estudo. Amostras CD-354, 355, 356ae 319. Outras ocorrências. Formação Santana, bacia do Araripe.

Discussão. Os espécimes de Harbinia aff. H. symmetrica (Krommelbein \& Weber, 1971) assemelham-se à espécie-tipo, embora apresentem a região anterior mais arredondada,-ângulo ântero-dorsal menos proeminente, além dos ângulos ântero e póstero-ventral um pouco mais acentuados, dando uma forma mais ovalada à espécie.

\section{Harbinia salitrensis (Krommelbein \& Weber, 1971)} (Figuras 4Q-T)

Hourcqia angulata salitrensis Krommelbein \& Weber, 1971:37-38, est. 6; figs. 26a, b, c.

Pattersoncypris cf. angulata salitrensis Silva-Telles \& Viana, 1990:319-321, est. 2, fig. 8.

Hourcqia? sp.2 Silva-Telles \& Viana, 1990:319-321, est. 3, figs. 5-6. Cultella sp. Depeche, Berthou \& Campos, 1990:305-306, pl. 2, fig. 3 .

Localidade tipo. Saco do Salitre, Estado do Pernambuco, Aptiano, Formação Santana, bacia do Araripe, Brasil.

Espécimes figurados. MPEG-46-MO a MPEG-49-MO.

Dimensões. MPEG-46-MO, carapaça, adulto, valva esquerda,

juvenil, MPEG-38-MO; J, VD, MPEG-39-MO; K, VE, juvenil, MPEG-40-MO; L, vista dorsal, juvenil, MPEG-41-MO. M-P, Carapaças de Harbinia sp. aff. H. symmetrica: M, VE, juvenil, MPEG-42-MO; N, VD, MPEG-43-MO; O, VE, juvenil, MPEG-44-MO; P, vista dorsal, juvenil, MPEG-45-MO. Q-T, Carapaças de Harbinia salitrensis: Q, VE, MPEG-46-MO; R, VD, juvenil, MPEG-47-MO; S, VE, juvenil, MPEG-48-MO; T, vista dorsal, juvenil, MPEG-49-MO. U-Y, Carapaças de Harbinia sp.: U, VE, MPEG-50-MO; V, VE, juvenil, MPEG-51-MO; W, VD, MPEG-52MO; X, vista dorsal, juvenil, MPEG-53-MO; Y, VD, MPEG-50-MO. Z-Z", Carapaças de Candona sp.: Z, VE, MPEG-54-MO; Z', VD, juvenil, MPEG-55-MO; Z", vista dorsal, MPEG-56-MO. Abreviações: VE, valva esquerda; VD, valva direita. Escala $=100 \mu \mathrm{m}$.

Figure 4. Ostracods from Grajaú Basin, Codó Formation. A-D, Carapaces of Harbinia micropapillosa: A, LV, MPEG-31-MO; B, RV, MPEG31-MO; C, LV, juvenile, MPEG-32-MO; D, dorsal view, MPEG-33-MO). E-H, Carapaces of Harbinia angulata: E, LV, juvenile, MPEG-34-MO; F, RV, MPEG-35-MO; G, LV, juvenile, MPEG-36-MO; H, dorsal view, juvenile, MPEG-37-MO. I-L, Carapaces of Harbinia sinuata: I, LV, juvenile, MPEG-38-MO; J, RV, MPEG-39-MO; K, LV, juvenile, MPEG-40-MO; L, dorsal view, juvenile, MPEG-41-MO. M-P, Carapaces of Harbinia sp. aff. H. symmetrica: M, LV, juvenile, MPEG-42-MO; N, RV, MPEG-43-MO; O, LV, juvenile, MPEG-44-MO; P, dorsal view, juvenile, MPEG-45-MO. Q-T, Carapaces of Harbinia salitrensis: Q, LV, MPEG-46-MO; R, RV, juvenile, MPEG-47-MO; S, LV, juvenile, MPEG-48-MO; T, dorsal view, juvenile, MPEG-49-MO. U-Y, Carapaces of Harbinia sp.: U, LV, MPEG-50-MO; V, LV, juvenile, MPEG-51-MO; W, RV, MPEG52-MO; X, dorsal view, juvenile, MPEG-53-MO; Y, RV, MPEG-50-MO. Z-Z"', Carapaces of Candona sp.: Z, LV , MPEG-54-MO; Z', RV, juvenile, MPEG-55-MO; Z", dorsal view, MPEG-56-MO. Abbreviations: LV, left valve; RV, right valve. Scale bar $=100 \mu \mathrm{m}$. 
comprimento: 0,957 mm; altura: 0,622 mm; MPEG-47-MO, carapaça, juvenil, valva direita, comprimento: $0,852 \mathrm{~mm}$; altura: 0,520 mm; MPEG-48-MO carapaça, juvenil, valva esquerda, comprimento: 0,832 mm; altura: 0,540 mm; MPEG-49-MO, carapaça, juvenil valva esquerda, comprimento: $0,753 \mathrm{~mm}$; largura: $0,411 \mathrm{~mm}$.

Localidade. Mina de Santo Amaro, Município de Codó, Estado do Maranhão.

Horizonte. Amostra D-319, Aptiano Superior, Formação Codó, bacia de Grajaú.

Material. Raro (aproximadamente 20 espécimes).

Ocorrência na área de estudo. Amostras CD-319 e 356a.

Outras ocorrências. Formação Santana, bacia do Araripe.

Discussão. Os espécimes encontrados na área de estudo são muito semelhantes à espécie-tipo descrita por Krommelbein \& Weber (1971), embora sejam menores.

\section{Harbinia sp.}

(Figuras 4U-Y)

Espécimes figurados. MPEG-50-MO a MPEG-53-MO.

Dimensões. MPEG-50-MO, carapaça, adulto, valva esquerda, comprimento: 0,840 mm; altura: 0,529 mm; MPEG-51-MO, valva esquerda, juvenil, comprimento: $0,623 \mathrm{~mm}$; altura: 0,439 mm; MPEG-52-MO carapaça, adulto, valva esquerda, comprimento: 0,841 mm; altura: 0,574 mm; MPEG-53-MO, carapaça, juvenil, valva esquerda, comprimento: $0,838 \mathrm{~mm}$; largura: $0,422 \mathrm{~mm}$.

Localidade. Mina de Santo Amaro, Município de Codó, Estado do Maranhão.

Horizonte. Amostra D-26, Aptiano Superior, Formação Codó, bacia de Grajaú.

Material. Comum (aproximadamente 50 espécimes).

Ocorrência na área de estudo. Amostra D-26 e 356a.

Outras ocorrências. Formação Santana, bacia do Araripe.

Discussão. Harbinia sp. difere das demais espécies do mesmo gênero por apresentar uma forma mais retangular. Embora esta espécie apresente um número razoável de espécimes, a maioria encontra-se muito mal preservados e quebrados sendo, assim, mantida, no presente trabalho, em nomenclatura aberta.

\author{
Subfamília CANDONINAE Kaufmann, 1900 \\ Candona Baird, 1845 \\ Candona sp. \\ (Figuras 4Z-Z”)
}

Ostracode 207 (Weber, 1964). Brito et al., 1984:214, est. s/n., figs. 1-3.

Ostracodes indetermines Depeche, Berthou \& Campos, 1990:303-304, pl. 1, figs. 3-4.

Gen. indet. sp. aff. 207 Silva-Telles \& Viana, 1990:309-327, est. 2, figs. 1,3 .

Gen. indet. sp. 207 Silva-Telles \& Viana, 1990:309-327, est. 2, fig. 2. Espécimes figurados. MPEG-54-MO a MPEG-56-MO.

Dimensões. MPEG-54-MO, carapaça, adulto, valva esquerda, comprimento: 0,799 $\mathrm{mm}$; altura: 0,396 mm; MPEG-55-MO, carapaça, juvenil, valva direita, comprimento: $0,708 \mathrm{~mm}$; altura:
0,362 mm; MPEG-56-MO, carapaça, adulto, vista dorsal, comprimento: $0,778 \mathrm{~mm}$; largura: $0,304 \mathrm{~mm}$.

Localidade. Mina de Santo Amaro, Município de Codó, Estado do Maranhão.

Horizonte. Amostra D-22, Aptiano Superior, Formação Codó, bacia de Grajaú.

Material. Muito abundante (aproximadamente 400 espécimes). Ocorrência na área de estudo. Amostras CD-354, 355, 356a, 318, 319 e D-22.

Outra ocorrência. Formação Santana (bacia do Araripe).

\section{DISTRIBUIÇÃO ESTRATIGRÁFICA DOS OSTRACODES}

A distribuição estratigráfica dos ostracodes nas sequiências sedimentares estudadas revelou que as assembléias faunísticas se diferenciam, ao longo dos perfis analisados, na diversidade, na abundância e na freqüência, acompanhando, assim, as três diferentes associações faciológicas definidas anteriormente para a área de estudo (Paz \& Rossetti 2001, 2005), e atribuídas a ambientes lacustres central, transicional e marginal.

Os níveis atribuídos a ambiente lacustre central, localizados na base da sequiência estudada e caracterizados por folhelhos negros betuminosos e evaporitos (Figura 3) apresentam-se, em geral, com grande volume de carapaças de ostracodes completas, embora deformadas, o que impossibilita sua identificação (amostras D-01, CD-354 e 355). As poucas carapaças inteiras recuperadas foram identificadas como Harbinia aff. $H$. symmetrica e Candona sp. (Figura 3). No topo do perfil (amostra D-26), níveis com as mesmas características de depósitos de lago central, mas que registram deposição sob condições oxidantes, mostram predomínio de Harbinia sp., associada a $H$. angulata. As valvas são, na maioria, isoladas, sendo algumas quebradas e, por vezes, deformadas, embora ocorram valvas e carapaças com a ornamentação bem preservada (Figura 3).

Os níveis atribuídos a ambiente lacustre transicional, posicionam-se na porção mediana da sequiência e contêm um volume significativamente maior de ostracodes mais bem preservados, se comparado ao de lago central. Os ostracodes (amostras D-11 e CD-356a) concentram-se em alguns horizontes onde houve intensa deposição carbonática e a formação de mudstone a packstone/grainstone ostracodal (Paz \& Rossetti 2001, 2005). Apesar de ainda se verificarem carapaças deformadas, existe um aumento notável no aparecimento de carapaças inteiras, dominantemente articuladas nestes depósitos carbonáticos, às vezes substituídas por calcedônia. Além disso, a assembléia de ostracodes presente nestes horizontes é mais diversificada, constituída por $H$. micropapillosa, $H$. sinuata, $H$. symmetrica, $H$. salitrensis e Harbinia sp., associadas a Candona sp. (Figura 3), todas apresentando distintos estágios ontogenéticos.

A ocorrência de ostracodes é mais freqüente e as assembléias faunísticas são mais numerosas nos níveis associados a ambiente lacustre marginal, presentes no topo dos perfis estudados (amostras D-20, CD 318, CD-319, D-22, D-24), embora menos diversificadas, inclusive com níveis monoespecíficos. Como descrito previamente, estes estratos contêm um volume maior de litologias carbonáticas em relação 
aos depósitos de lago central e transicional, representadas por calcário ostracodal, calcário peloidal, ritmito calcário/ folhelho (Paz \& Rossetti 2001, 2005). Os calcários ostracodais (amostra CD-318) estão representados sob forma de concreções com até $30 \mathrm{~cm}$ de diâmetro e contêm uma paleofauna monoespecífica de $H$. micropapillosa com grande abundância de carapaças fechadas e bem preservadas, evidenciando diferentes estágios ontogenéticos. Assembléias monoespecíficas foram também registradas em outros dois níveis, principalmente em associação com calcários peloidais (packstone ooidal/pisoidal) e com ritmitos de calcário/folhelho, onde são representadas por Candona sp. (amostras D-22) e $H$. angulata (amostra D-24). Nestas amostras, existe domínio de carapaças fechadas bem preservadas, também mostrando diferentes estágios ontogenéticos. Ainda, nos depósitos de lago marginal, dois outros horizonte de calcário peloidal (CD319 e D-20) constituem-se de uma ostrafauna um pouco mais diversificada representada por Harbinia sp. aff. H. symmetrica associada a $H$. salitrensis e a Candona sp., mas dominada por fragmentos mal preservados (deformados e ferruginizados), provavelmente em consequiência de fatores diagenéticos.

\section{DISCUSSÃO}

O estudo dos ostracodes da Formação Codó nos perfis estudados revelou a ocorrência exclusiva de Harbinia e Candona, gêneros típicos de ambientes não marinhos lacustres. A presença dominante de espécies do gênero Harbinia em um ambiente deposicional lacustre hipersalino e de baixa disponibilidade de oxigênio, como interpretado para a área de estudo por Paz \& Rossetti (2005) é explicável, uma vez que este gênero tem sido registrado previamente em ambientes com condições ambientais semelhantes, na Formação Santana (Arai \& Coimbra, 1990; SilvaTelles \& Viana, 1990; do Carmo et al., 1999, Coimbra et al, 2002). Nas fases características dos depósitos de lago marginal, caracterizadas como de condições ambientais de extrema exposição subaérea, a ocorrência de amostras com ostracodes é mais frequiente e as assembléias são mais abundantes. A maior frequiência e a ocorrência de níveis monoespecíficos de $H$. micropapillosa, H. angulata e de Candona sp., nessa fácies devese, provavelmente, à resistência destas espécies às condições ambientais extremas que se desenvolveram em função de um processo cíclico "rápido" de contração, seguido de expansão do sistema. A adaptação e a resistência dessas espécies às mudanças ambientais bruscas ocorridas durante a fase de contração do lago favoreceram o rápido desenvolvimento das populações, em um primeiro momento. O retorno das condições de expansão do lago trouxe consigo o fenômeno de mortandade em massa.

O modelo paleoambiental aqui apresentado condiz com modelos cíclicos já apresentados por Silva-Telles \& Viana (1990) a partir do estudo de ostracodes da Formação Santana (bacia do Araripe). Segundo os autores, eventos de expansão das comunidades, seguidos por mortandade em massa, estariam associados, na maioria das vezes, a episódios cíclicos de oxigenação dentro de um sistema lacustre dominado por baixa disponibilidade de oxigênio, como o que foi interpretado para a área de estudo.
A assembléia de ostracodes registrada nos estratos estudados permitiu, ainda, confirmar que a deposição da Formação Codó, na área de estudo, ocorreu durante o intervalo Aptiano. A fauna estudada apresenta grande similaridade, principalmente, com ostracodes não-marinhos registrados em outras unidades cretáceas do intervalo Aptiano, como a Formação Santana (Depeche et al., 1990; Silva-Telles \& Viana, 1990; Berthou et al., 1993, Coimbra et al., 2002), a Formação Alagamar (bacia Potiguar, do Carmo, 1998, do Carmo et al., 1999), a Formação Riachuelo (bacia de Sergipe-Alagoas, Krömmelbein \& Weber, 1971) e a Formação Quiricó (bacia Sanfranciscana, do Carmo et al., 2004). A ocorrência das espécies-guias $H$. angulata, $H$. sinuata e Candona sp. (Ostracode sp. 207), associada aos registros de Sergipea variverrucata, permitiram confirmar intervalo Neoaptiano para os depósitos estudados, conforme Paz (2005) e Rossetti et al. (2000).

\section{CONCLUSÕES}

A fauna de ostracodes presente nos depósitos lacustres neoaptianos da Formação Codó expostos na borda leste da bacia de Grajaú permitiu o reconhecimento de Harbinia micropapillosa, $H$. angulata, $H$. sinuata, $H$. sp. aff. $H$. symmetrica, $H$. salitrensis, Harbinia sp. e Candona sp. O estudo das paleocomunidades de ostracodes nos perfis estudados suporta os resultados das interpretações paleoambientais feitos anteriormente, a partir de dados faciológicos (Paz \& Rossetti, 2001, 2005; Rossetti \& Góes, 2000; Paz, 2005), para a área de estudo. A existência de uma ciclicidade dentro de um ambiente deposicional lacustre, com variação no teor de oxigênio e da salinidade, associadas a eventos de expansão e de contração do lago estão expressos na distribuição da ostracofauna. Tais eventos parecem ter sido suficientemente rápidos e de curta duração, gerando desenvolvimento populacional exclusivo de algumas espécies seguido de mortandade em massa. A assembléia de ostracodes registrada nos estratos estudados permitiu, ainda, confirmar que a deposição da Formação Codó na área de estudo ocorreu durante o intervalo Neoaptiano, sob domínio de condições continentais lacustres.

\section{AGRADECIMENTOS}

Ao CNPq, pelo apoio financeiro (proc. 460252/01), a Hilton Túlio Costi (LME/MPEG), pelo auxílio na obtenção das fotografias no microscópio eletrônico de varredura, a Denys José Xavier Ferreira, pela edição das imagens da Figura 4 e aos revisores pelas minuciosas correções e sugestões deste trabalho. Este trabalho foi uma contribuição ao XIX Congresso Brasileiro de Paleontologia/VI Congresso Latino-americano de Paleontologia, ocorrido em Aracajú, SE, em agosto de 2005.

\section{REFERÊNCIAS}

Arai, M. \& Coimbra, J.C. 1990. Análise paleoecológica do registro das primeiras ingressões marinhas na Formação Santana 
(Cretácio Inferior da Chapada do Araripe). In: SIMPÓSIO SOBRE A BACI DO ARARIPE E AS BACIAS INTERIORES DO NORDESTE, 1, 1990. Atas, Crato, URCA, p. 225-239.

Azevedo, R.P. 1991. Tectonic evolution of Brazilian equatorial margin basins. University of London, Tese de Doutorado, $445 \mathrm{p}$.

Bate, R.H. 1972. Phosphatized ostracodes with appendages from the Lower Cretaceous of Brazil. Palaeontology, 15(3):379-393.

Bate R.H. 1973. On Pattersoncypris micropapillosa Bate. In: P.C. Sylvester Bradley \& D. J. Silveter (eds.) Stereo atlas of ostracode shells. Leicester, University of Leicester, Department of Geology, 1973, 1(2):101-108.

Berthou, P.Y.; Depeche, F.; Colin, J.P.; Melo Filgueira, J.B. \& Lopes Teles, M.S. 1993. Some new ostracodes and palynomorphs from the Rio Batateira and Crato lithologic unit (Upper AptianLowermost Albian) of the Araripe Basin (NE Brazil). In: CONGRESSO BRASILEIRO DE PALEONTOLOGIA, 13, 1993. Boletim de Resumos, CPRM, p. 176.

Brito, H.S.; Viana, C.F. \& Praça, U.M. 1984. Atlas dos ostracodes não-marinhos do Brasil (Neojurássico-Eocretáceo). Rio de Janeiro, Petrobrás, 214 p.

do Carmo, D.A. 1998. Taxonomia, paleoecologia e distribuição estratigráfica dos ostracodes da Formação Alagamar (Cretáceo Inferior), bacia Potiguar, Brasil. Programa de Pós-Graduação em Geociências, Universidade Federal do Rio Grande do Sul, Tese de Doutorado, 156 p.

do Carmo, D.A.; Sanguinetti, Y.T.; Coimbra, J.C. \& Guimarães, E.M. 1999. Paleoecologia dos ostracodes não-marinhos do Cretáceo Inferior da bacia Potiguar, RN, Brasil. In: SIMPÓSIO SOBRE O CRETÁCEO DO BRASIL, 5, 1999. Boletim, Campus de Rio Claro, UNESP, p. 383-391.

do Carmo, D.A.; Tomassi, H.Z. \& Oliveira S.B.S.G. 2004. Taxonomia e distribuição estratigráfica dos ostracodes da Formação Quiricó, Grupo Areado (Cretáceo Inferior) bacia Sanfranciscana, Brasil. Revista Brasileira de Paleontologia, 7(2):139-149.

Coimbra, J.C.; Arai, M. \& Carreño, A.L. 2002. Biostratigraphy of Lower Cretaceous microfossils from the Araripe Basin, northeastern Brazil. Geobios, 35(6):687-698.

Depeche, F.; Berthou, P.Y. \& Campos, D.A. 1990. Quelques observations sur les faunes d' ostracodes du Crétacé du Bassin d'Araripe (N.E. du Brésil). In: SIMPÓSIO SOBRE A BACIA DO ARARIPE E BACIAS INTERIORES DO NORDESTE, 1, 1990. Atas, Crato, URCA, p. 293-308.

Góes, A.M. \& Coimbra, A.M. 1996. As bacias sedimentares da Província Sedimentar do Meio-Norte. In: SIMPÓSIO DE GEOLOGIA DA AMAZÔNIA, 5, 1996. Boletim de Resumos Expandidos, Belém, SBG, p. 186-187.

Góes, A.M. \& Rossetti, D.F. 2001. Gênese da bacia de São LuísGrajaú, meio norte do Brasil. In: D.F. Rossetti; A.M. Góes \& W. Truckenbrodt (eds.) O Cretáceo da bacia de São LuísGrajaú. Belém, Coleção Friedrich Katzer, Museu Paraense Emílio Goeldi, p. 15-31.

Krömmelbein, K. \& Weber, R. 1971. Ostracoden des "NordostBrasilianischen Wealden". Geologisches Jahrbuch, 115:1-93.

Miranda, M.C.C. 2005. Fácies, petrografia e paleontologia de depósitos albianos do Grupo Itapecuru, área de Coroatá (MA), bacia do Grajaú. Programa de Pós-graduação em Geologia e Geoquímica, Universidade Federal do Pará, Dissertação de Mestrado, 139 p.

Moore, R.C. 1961.Treatise on Invertebrate Paleontology. Part Q. Arthropoda 3 Crustacea-Ostracoda. Lawrence, Geological Society of America and University of Kansas Press, 442 p.

Paz, J.D.S. 2005. Fácies, petrografia e geoquímica da Formação
Codó, Neoaptiano, bacia de São Luís-Grajaú. Programa de Pós-graduação em Geologia e Geoquímica, Universidade Federal do Pará, Tese de Doutorado, 148 p.

Paz, J.D.S. \& Rossetti, D.F. 2001. Reconstrução paleoambiental da Formação Codó (Aptiano), borda leste da bacia do Grajaú, MA. In: D.F. Rossetti, A.M.; Góes \& W. Truckenbrodt (eds.) O Cretáceo na bacia de São Luís-Grajaú, Belém, Coleção Friedrich Katzer, Museu Paraense Emílio Goeldi, p. 77-100.

Paz, J.D.S. \& Rossetti, D.F. 2005. Linking lacustrine cycles with syn-sedimentary tectonic episodes: an example from the Codó Formation (late Aptian), northeastern Brazil. Geology Magazine, 142(3):269-285.

Rossetti, D.F. 1997a. Facies analysis of the lower succession of the upper Itapecurú Formation, São Luís Basin, northern Brazil. In: M.L. Costa \& R. Angelica (eds.) Contribuições à geologia da Amazônia. Belém, Ed. Falângola, p. 241-284.

Rossetti, D.F. 1997b. Sedimentology and sequential analysis of Cenomanian to early Tertiary (?) deposits in northern Brazil. In: SIMPÓSIO LATINOAMERICANO DE SEDIMENTOLOGIA, 1, 1997. Boletim de Resumos Expandidos, Isla Margarita, Venezuela, 2:233-237.

Rossetti, D.F. 1998. Facies architecture and sequential evolution of incised valley estuarine fills: the Upper Itapecurú Formation (São Luís Basin), northern Brazil. Journal of Sedimentary Research, 68:299-310.

Rossetti, D.F. 2000. Influence of low amplitude/high frequency relative sea-level changes in a wave-dominated estuary (Miocene), São Luís Basin, northern Brazil. Sedimentary Geology, 133:295-324.

Rossetti, D.F. 2001. Arquitetura deposicional da bacia de São LuísGrajaú. In: D.F. Rossetti; A.M. Góes \& W. Truckenbrodt (eds.) O Cretáceo na bacia de São Luís-Grajaú, Belém, Coleção Friedrich Katzer, Museu Paraense Emílio Goeldi, p. 31-46.

Rossetti, D.F. \& Góes, A.M. 2000. Deciphering the sedimentological imprint of paleoseismic events: an example from the Aptian Codó Formation, northern Brazil. Sedimentary Geology, 235:137-156.

Rossetti, D.F.; Góes, A.M. \& Paz, J.D.S. 2004. Facies analysis of the Codó Formation (Late Aptian) in the Grajaú Area, Southern São Luís-Grajaú Basin. Anais da Academia Brasileira de Ciências, 76(4):791-806.

Rossetti, D.F.; Góes, A.M.; Paz, J.S. \& Macambira, M. 2000. Sequential analysis of the Aptian deposits from the São Luís and Grajaú basins, Maranhão State (Brazil) and its implication for unraveling the origin of evaporites. Revista Brasileira de Geociências, 30(3):466-469.

Rossetti, D.F. \& Truckenbrodt, W. 1997. Revisão estratigráfica para os depósitos do Albiano-Terciário Inferior (?) na bacia de São Luís, Maranhão. Boletim do Museu Paraense Emílio Goeldi, 9:29-41 (Série Ciências da Terra).

Silva-Telles, Jr., A.C. \& Viana, M.S.S. 1990. Paleoecologia dos ostracodes da Formação Santana (bacia do Araripe): um estudo ontogenético de populações. In: SIMPÓSIO SOBRE A BACIA DO ARARIPE E BACIAS INTERIORES DO NORDESTE, 1, 1989, Crato. Atas, DNPM, p. 309-328.

Viana, M.S.S.; Brito, P.M. \& Silva-Telles Jr., A.C. 1989. Paleontologia de uma camada de folhelhos pirobetuminosos do Membro Romualdo, Formação Santana, na Mina Pedra Branca, Município de Nova Olinda, Ceará. In: CONGRESSO BRASILEIRO DE PALEONTOLOGIA, 11, 1989. Anais, Curitiba, SBP, p. 207-217.

Received in October, 2005; accepted in October, 2006 